\title{
Optimal primary end point in Phase II trials of immune checkpoint inhibitors for advanced solid cancers: an evolving issue
}

\author{
Giandomenico Roviello*,1, Daniele Generali',3 \& Anna lanza ${ }^{4}$ \\ ${ }^{1}$ Translational Oncology Unit, Department of Health Sciences, University of Florence, viale Pieraccini, 6, 50139 Florence, Italy \\ ${ }^{2}$ Department of Medical, Surgery \& Health Sciences, University of Trieste, Trieste, Italy \\ ${ }^{3}$ Breast Cancer Unit \& Translational Research Unit, ASST Cremona, Cremona, Italy \\ ${ }^{4}$ Department of Medical, Surgery \& Health Sciences, University of Trieste, Piazza Ospitale 1, 34129 Trieste, Italy \\ *Author for correspondence: giandomenicoroviello@hotmail.it
}

"ICls have revolutionized the treatment scenario of several solid tumors. However, there is urgent need to identify dedicated end points in early clinical Phase II studies with ICls. This identification could better and more cost-effectively drive the selection of the most accurate agent to be used in randomized Phase III trials. "

First draft submitted: 12 December 2017; Accepted for publication: 21 January 2019; Published online: 21 February 2019

Keywords: end points • immunotherapy • PD-1 • PD-L1 • Phase II

Immune checkpoint inhibitors (ICIs) are a novel class of therapeutic agents whose peculiar mechanism of action, based on an activation of the immune system against tumor cells, has changed the management of several advanced solid tumors [1]. The use of the immune system against tumors is not a novelty in oncology field; in fact, IL-2, a cytokine produced by activated cytotoxic T lymphocytes, has shown a certain antitumor activity in melanoma and renal cell carcinoma but also in other solid tumors [2]. In addition, interferon (IFN) is another cytokine that has achieved US FDA approval as a single agent for the adjuvant therapy of stage III melanoma [3]. Finally, it has been suggested that response to chemotherapy and radiotherapy involves T-cell activation, corroborating the hypothesis that combining conventional chemotherapy with blocking of immune checkpoint would lead to an improvement in tumor response [3]. The evolution of the strategy of treatment that was started with the use of IL-2 and IFN lead to the development of the novel class of anticancer drugs known as ICIs. Ipilimumab was the first human monoclonal antibody that targets the cytotoxic $T$ lymphocyte-associated antigen 4 receptor that showed an efficacy on advanced melanoma [4]. Consequently, the discovery of the antigen programmed cell death 1 (PD-1), a protein expressed on the surface of activated T cells, and its ligand PD-L1 (also known as B7-H1) expressed mainly in tumor cells as well as other immune cells, had led to a development of novel classes of ICIs: the anti-PD-1 and anti-PD-L1 antibodies that work by blocking the PD-1/PD-L1 pathway. The PD1 binding of its ligand PD-L1 results in inhibition of T-cells proliferation, so that anti-PD-1 or PD-L1 agents prevent this binding and consequently potentiate T-cell activity, including antitumor response. To date, two anti-PD-1 antibodies (nivolumab and pembrolizumab) have been approved for several solid tumors [5] and it is of September 2018 the news of cemiplimab US FDA approval for cutaneous squamous cell carcinoma. On the other hand, the anti-PD-L1s atezolizumab, avelumab and durvalumab are approved or under investigation [5]. Interestingly, the first approved ICI, ipilimumab, to date has been used only in melanoma or renal cell carcinoma [6]. An important point in favor of ICIs is the different toxicity profile compared with common chemotherapy, resulting in good compliance even for patients with advanced diseases. Finally, ICIs have been investigated in combination with chemotherapy in non-small-cell lung cancer [7] and with antiangiogenic agents in renal cell carcinoma [8].

When a novel agent is approved in the field of oncology, there are generally two main issues. The first is identification of a surrogate marker for survival, required to select the appropriate primary end point for the early and further phases of drug development. This option permits quicker and less expensive studies. The second issue is the need of selective biomarkers to better tailor the therapeutic agent. For example, the guideline for decreasing 
of $50 \%$ of more of the serum prostate-specific antigen value in patients with prostate cancer is usually used as a valid surrogate for survival and to identify in an earlier phase of treatment those patients who are not responsive to anticancer agents and who may benefit from a change in therapy $[9,10]$. For what concerns ICI treatments, the role of PD-L1 expression as a marker of response remains controversial [11], although it has been used as a selective criterion for pembrolizumab treatment of patients with non-small-cell lung cancer. In general, the clinical evaluation of the activity and efficacy of a conventional or biological cancer treatment is assessed by change in tumor burden. The main criteria used for the evaluation of these changes are the Response Evaluation Criteria in Solid Tumors (RECIST), which are used not only in experimental but also in clinical practice as a guide to continue or discontinue a treatment [12]. According to the RECIST criteria, the rate of total patients with a complete response or a partial response defines the objective tumor response rate (ORR). The ORR is generally used as primary end point in Phase II trials, hence the choice of ORR as the preferred surrogate parameter for overall survival (OS). The Phase II trial is generally the first step to evaluate the efficacy of an anticancer agent. Based on this universal approach for drug development, the design of a typical Phase II trial in immunotherapy is single arm with a moderate number of patients enrolled, with ORR as the primary end point. Unfortunately, researchers were faced with a unique event that characterizes the pattern of response and progression of ICIs compared with those of conventional chemotherapy or biological/molecular-targeted therapies: the pseudo-progression. This event is characterized by the development of new lesions or an increase in existing lesions, which is not strictly related to a resistance to treatment [13] but rather to an infiltration of immune cells triggered by ICIs [14,15]. As matter of fact, the correlation between ORR and survival in Phase II ICI trial seems to be weakened by pseudo-progression, an expected event in this kind of therapy, and by the lack of a careful selection of the patient. Indeed, a more accurate selection method would better determine an ulterior lowering of the numbers; on the other hand, it should also discriminate a greater effectiveness of agents. In this context, a recent meta-analysis [16] compared the primary end points of 87 Phase II trials, weighing the strength of the correlation of the surrogate parameter with 6 months progression-free survival (PFS) and 12-month OS rate using a unique prediction model of correlation. They found that $60 \%$ of Phase II trials had ORR as the primary end point, but that ORR correlated poorly with OS. However, 6-month PFS correlates relatively strongly with the 12-month OS rate and is proposed as a preferred parameter to be used in Phase II studies. In addition, Kaufman $e t$ al. performed a systematic literature review with meta-analysis to assess the correlation of classical clinical end points used in clinical trial such as PFS, ORR or disease control rate and their use for surrogacy of OS. A total of 27 randomized controlled trials were evaluated for a total of more than 10,000 patients. The rate of patients with tumor response and PFS hazard ratio (HR) showed a weak correlation with survival; however, 6-month PFS HR was predictive of survival. Interestingly, PFS HR at 6 months was highly predictive of survival in the subgroup of patients treated with single-agent ICI, but was weakly predictive in the pooled analysis. In line with the two above literature analysis [16,17], a recent robust meta-regression of 24 trials, for a total of 11,894 patients, aims to assess the role of the tumor response rate (RR) after ICI-based therapy as a potential surrogate end point of PFS and OS confirmed a weak correlation between RR and OS [5].

To try to avoid the limitations of ORR during ICI-based therapy, a modified version of the RECIST criteria has been recently proposed, immune-related response criteria (iRECIST) and immune-modified response criteria (imRECIST), in response to the need for an appropriate scale to evaluate and distinguish pseudo-progression from real progression of disease [18]. Nevertheless, only $6 \%$ of the included trials considered iRECIST, either as a primary or secondary end point [16]. The recurrence rate of pseudo-progressions in ICI-treated tumor strictly depends on the scale used to measure the response, and onset occurs and is completely concluded on average in a 1-year period [19].

All these data underscore the limitations of Phase II trials and the challenges involved in translating early promising clinical results from Phase II trials in efficacy to survival of Phase III studies. In fact, promising Phase II trials have not translated into successful Phase III trials for some ICIs [20]. Nevertheless, further studies are ongoing to explore the role of ICIs in combination with chemotherapy or antiangiogenic agents. For these types of studies, the optimal primary end points in early clinical investigations are not well defined. Although it could be speculated that potential future end points of Phase II studies for ICIs may include classical clinical end points in combination with newer end points such as stability in overall tumor burden, sustained reduction or durable response rate, to date, very few Phase II studies have used this combination of end points and further investigations are required to define its role. 


\section{Conclusion}

ICIs have revolutionized the treatment scenario of several solid tumors. However, there is urgent need to identify dedicated end points in early clinical Phase II studies with ICIs. This identification could better and more costeffectively drive the selection of the most accurate agent to be used in randomized Phase III trials. At this time point, it seems that the phenomenon of false progression and the difficulties in selecting and finding the appropriate population of patients indirectly affect the further approval of compounds for the successive phases of research. Considering that ORR is the most used surrogate end point and that correlates poorly with survival, other end points such as 6-month PFS could be used as surrogate markers for survival in future Phase II ICI trials.

\section{Acknowledgments}

Authors thank Elsevier Language Editing Services for the English editing of this manuscript.

\section{Financial \& competing interests disclosures}

The authors have no relevant affiliations or financial involvement with any organization or entity with a financial interest in or financial conflict with the subject matter or materials discussed in the manuscript. This includes employment, consultancies, honoraria, stock ownership or options, expert testimony, grants or patents received or pending, or royalties.

Medical writing support was provided by Elsevier Language Editing Services and was self-funded by authors.

\section{References}

1. Wolchok JD, Kluger H, Callahan MK et al. Nivolumab plus ipilimumab in advanced melanoma. N. Engl. J. Med. 11; 369(2), 122-133 (2013).

2. Roviello G, Zanotti L, Correale P et al. Is still there a role for IL-2 for solid tumors other than melanoma or renal cancer? Immunotherapy 9(1), 25-32 (2017).

3. Lee S, Margolin K. Cytokines in cancer immunotherapy. Cancers 133(4), 3856-3893 (2011).

4. Tarhini AA, Iqbal F. CTLA-4 blockade: therapeutic potential in cancer treatments. Onco Targets Ther. 24(3), 15-25 (2010).

5. Roviello G, Andre F, Venturini $S$ et al. Response rate as a potential surrogate for survival and efficacy in patients treated with novel immune checkpoint inhibitors: a meta-regression of randomised prospective studies. Eur. J. Cancer 86, 257-265 (2017).

6. Martin-Liberal J, Ochoa de Olza M, Hierro C, Gros A, Rodon J, Tabernero J. The expanding role of immunotherapy. Cancer Treat. Rev. 54, 74-86 (2017).

7. Zhang $\mathrm{Y}$, Zhou $\mathrm{H}$, Zhang $\mathrm{L}$. Which is the optimal immunotherapy for advanced squamous non-small-cell lung cancer in combination with chemotherapy: anti-PD-1 or anti-PD-L1? J. Immunother. Cancer 6(1), 135 (2018)

8. Bracarda S, Porta C, Sabbatini R, Rivoltini L. Angiogenic and immunological pathways in metastatic renal cell carcinoma: a counteracting paradigm or two faces of the same medal? The GIANUS review. Crit. Rev. Oncol. Hematol. S1040-S8428(18), 30141-0 (2018)

9. Roviello G. Role of PSA response as a marker for efficacy of patients with castration-resistant prostate cancer treated with novel hormonal therapies. Int. J. Biol. Mark. 33(1), 132-133 (2018).

10. Francini E, Petrioli R, Rossi G, Laera L, Roviello G. PSA response rate as a surrogate marker for median overall survival in docetaxel-based first-line treatments for patients with metastatic castration-resistant prostate cancer: an analysis of 22 trials. Tumour Biol. 35(11), 10601-10607 (2014).

11. Kluger HM, Zito CR, Turcu G et al. PD-L1 studies across tumor types, its differential expression and predictive value in patients treated with immune checkpoint inhibitors. Clin. Cancer Res. 23(15), 4270-4279 (2017).

12. Eisenhauer EA, Therasse P, Bogaerts J et al. New response evaluation criteria in solid tumours: revised RECIST guideline (version 1.1). Eur. J. Cancer 45, 228-247 (2009).

13. Chiou VL, Burotto M. Pseudoprogression and immune-related response in solid tumors. J. Clin. Oncol. 33(31), 3541-3543 (2015).

14. Wolchok JD, Hoos A, O'Day S et al. Guidelines for the evaluation of immune therapy activity in solid tumors: immune-related response criteria. Clin. Cancer Res. 15(23), 7412-7420 (2009)

15. Hodi FS, Butler M, Oble DA et al. Immunologic and clinical effects of antibody blockade of cytotoxic T lymphocyte-associated antigen 4 in previously vaccinated cancer patients. Proc. Natl Acad. Sci. USA 105(8), 3005-3010 (2008)

16. Ritchie G, Gasper H, Man J et al. Defining the most appropriate primary end point in Phase II trials of immune checkpoint inhibitors for advanced solid cancers: a systematic review and meta-analysis. JAMA Oncol. 4(4), 522-528 (2018)

17. Kaufman HL, Schwartz LH, William WN Jr et al. Evaluation of classical clinical end points as surrogates for overall survival in patients treated with immune checkpoint blockers: a systematic review and meta-analysis. J. Cancer Res. Clin. Oncol. 144(11), 2245-2261 (2018)

18. Seymour L, Bogaerts J, Perrone A et al. RECIST working group. iRECIST: guidelines for response criteria for use in trials testing immunotherapeutics. Lancet Oncol.18(3), e143-e152 (2017) 
19. Kurra V, Sullivan RJ, Gainor JF, Hodi FS, Gandhi L, Sadow CA. Pseudoprogression in cancer immunotherapy: rates, time course and patient outcomes. J. Clin. Oncol. 34(no. 15_suppl.), S6580-S6580 (2016)

20. Gopalakrishnan D, Koshkin VS, Ornstein MC, Papatsoris A, Grivas P. Immune checkpoint inhibitors in urothelial cancer: recent updates and future outlook. Ther. Clin. Risk Manag. 14, 1019-1040 (2018) 